\title{
Exposer le racisme. Exhibit B et le public oppositionnel
}

Exhibiting Racism. Exhibit B and the oppositional public

\section{Maxime Cervulle}

\section{(2) OpenEdition \\ Journals}

Édition électronique

URL : http://journals.openedition.org/edc/6775

DOI : $10.4000 /$ edc. 6775

ISSN : 2101-0366

Éditeur

Université de Lille

Édition imprimée

Date de publication : 1 juin 2017

Pagination : $37-54$

ISBN : $978-2-917562-17-8$

ISSN : $1270-6841$

Référence électronique

Maxime Cervulle, «Exposer le racisme. Exhibit $B$ et le public oppositionnel », Études de communication

[En ligne], 48 | 2017, mis en ligne le 01 juin 2017, consulté le 10 décembre 2020. URL : http:// journals.openedition.org/edc/6775 ; DOI : https://doi.org/10.4000/edc.6775 


\section{Exposer le racisme. Exhibit $B$ et le public oppositionnel} Exhibiting Racism. Exhibit B and the oppositional public 
Comment se distribue la légitimité à dire le racisme, selon quels critères de partages et en vertu de quelle conception de l'objet à propos duquel on cherche à s'exprimer ? La médiatisation de la controverse autour d'Exhibit B, installation-performance mise en scène par Brett Bailey et présentée en région parisienne à la fin de l'année 2014, constitue un cas intéressant pour esquisser quelques pistes de réponses. Présentée comme antiraciste, l'œuvre fut confrontée à une forte contestation menée par des collectifs dont l'antiracisme revendiqué se fonde sur une définition antagoniste. Toutefois, plutôt que de mettre en scène un conflit définitionnel, la presse nationale quotidienne qui se fait l'écho de cette controverse participe de la délégitimation de la contestation en renvoyant à la fois le public oppositionnel d'Exhibit $B$ à un excès politique et à une incompétence esthétique.

Mots-clés : racisme, publics, théâtre, représentation, presse.
How is legitimacy to speak of racism distributed? Media coverage of the controversy surrounding Exhibit B, an installation performance directed by Brett Bailey and presented in the Paris area in 2014, is an interesting case with which to consider some possible answers to this question. Although presented as anti-racist, Bailey's work elicited strong protests on the part of groups whose conception of anti-racism is antagonistic in nature. However, rather than staging a definitional conflict, the national daily press, in its coverage of this controversy, contributed to the delegitimization of the protest by casting the oppositional public of Exhibit B both as politically excessive and aesthetically incompetent.

Keywords: racism, public, theater, representation, press. 
On dit « on va voir... » mais voir quoi ? Pourquoi dois-je voir ? Que doisje voir qui va me faire comprendre que l'esclavage et le colonialisme étaient condamnables? Me montrer des mains coupées comme celles qui l'étaient sous Léopold II au Congo dans les exploitations de caoutchouc? Ce que je vois ? Je ne vois rien! Je vois une performance. J'interroge cette injonction à voir.

Françoise Vergès (Vergès et Mestre, 2017, 103).

Comment donner à voir le racisme? Quelles sont les conditions d'une représentation qui parvienne à en transmettre toute la violence, à la rendre sensible et tangible, sans pour autant la reproduire? Comme l'écrit Achille Mbembe $(2013,23)$, « de la race (ou du racisme), I'on ne peut parler que dans un langage fatalement imparfait, gris, voire inadéquat ". C'est toutefois à cette fatalité que doit se heurter tout discours critique qui tend à dire le refus du présent racial. $L$ 'installation-performance Exhibit $B$ du metteur en scène sud-africain Brett Bailey, et plus particulièrement la controverse à laquelle elle a donné lieu lors de sa programmation fin 2014 au Théâtre Gérard Philipe (TGP) de Saint-Denis et au Centquatre à Paris, constitue un cas intéressant pour emprunter cette voie de questionnement.

Présentée comme une œuvre antiraciste, Exhibit $B$ propose une déambulation parmi une série de douze tableaux vivants qui, en reconstituant des pans de l'histoire (post)coloniale, exposent la violence à laquelle les corps noirs ont été et sont soumis ${ }^{1}$. Les comédien·ne·s enchaîné·e·s, encagé·e·s ou bâillonné·e·s, immobiles et silencieux/cieuses en leurs décors, renvoient le regard que porte sur eux les spectateurs/trices. Pour Sabine Cessou (Libération, 8 décembre 2014), "la performance vise à retourner le procédé du 'zoo humain' à l'envoyeur, avec des acteurs noirs qui fixent le visiteur, soutiennent son regard et ne le lâchent pas des yeux ". L'œuvre suscite toutefois l'émoi de certains collectifs antiracistes : après avoir déclenché une vaste polémique à Londres, qui a abouti à la déprogrammation de la pièce par le Barbican en septembre 2014, une contestation se lève en région parisienne. De pétition en tracts, de billets de blogs en rassemblements, une mobilisation s'organise sous l'impulsion d'un collectif ad hoc, intitulé Contre Exhibit B, de la Brigade Anti-Négrophobie et, dans une moindre mesure, du Conseil représentatif des associations noires (CRAN). Celle-ci donne cependant à voir les divisions qui parcourent le champ antiraciste : des associations, comme le Mouvement contre le racisme et pour l'amitié entre les peuples (Mrap), la Ligue internationale contre le racisme et l'antisémitisme (Licra) ou la Ligue des droits de l'homme (LDH), s'insurgent contre les demandes d'annulation et défendent la nécessité de ce type d'œuvre². La controverse prend néanmoins une telle ampleur qu'elle

1 Pour une présentation détaillée de la pièce, voir Maire (2016).

2 Communiqué de la LDH, de la Licra et du Mrap, «Exhibit $B$ : un spectacle qui ne doit pas être interdit ou annulé ». Paris, 21 novembre 2014. 
suscite des prises de position de la maire de Paris et de la ministre de Culture en faveur de l'œuvre et de sa programmation, en particulier lorsque le collectif Contre Exhibit $B$ saisit en référé le tribunal administratif de Paris afin d'obtenir I'interdiction du spectacle pour « atteinte à la dignité humaine ${ }^{3}$.

La controverse autour d'Exhibit $B$ met en tension différentes formes d'antiracisme, chacune porteuse d'une conception du racisme et des moyens de lutte appropriés à cette conception. Dans cet article, je m'intéresserai particulièrement à la médiatisation de cette controverse, en partant d'un corpus de 41 articles publiés dans la presse nationale quotidienne. Constitué via Europresse, ce corpus comprend l'ensemble des articles contenant le nom « Exhibit B » publiés dans les quotidiens français entre le 27 novembre 2013 (à l'occasion de la première représentation d'Exhibit $B$ à Paris) et le 17 septembre 2015. Ils ont été publiés dans Aujourd'hui en France, La Croix, Le Figaro, L'Humanité, Libération et Le Monde, mais c'est avant tout dans la presse de gauche et centre-gauche que se déploie la controverse ${ }^{4}$. La médiatisation de l'œuvre et de la controverse qu'elle suscite se déroule essentiellement dans les pages «Culture » des quotidiens, et une part conséquente des journalistes qui y prennent part sont spécialisé·e·s dans le journalisme culturel, voire dans la critique de théâtre. Les articles se répartissent à parts égales en trois principales catégories : des reportages, qui relatent les manifestations contre l'œuvre; des tribunes, avant tout écrites par des universitaires et des artistes ; et enfin, des comptes-rendus de l'installation-performance ou des portraits de Brett Bailey présentant sa trajectoire biographique et artistique.

L'analyse de ce corpus a pour but d'interroger les dynamiques de définition conflictuelle de l'antiracisme dans la presse quotidienne nationale, saisie comme une arène de discours spécifique dotée d'un fort pouvoir de dé/légitimation et qui, à ce titre, participe de la redéfinition des contours des publics. Si l'on comprend le public comme étant lui-même " un espace discursif [...] qui existe grâce à l'adresse qui lui est faite " (Warner, 2014, 67, mes italiques), on peut s'interroger sur la manière dont la presse participe de la formation du public imaginé d'Exhibit $B$, mais surtout de la façon dont, en représentant les manifestant·e.s et pétitionnaires mobilisé·e.s contre l'œuvre, elle contribue à les reconnaître ou non comme public, et donc à les rendre plus ou moins susceptibles de faire entendre des revendications. La question de la distribution de la légitimité à dire le racisme recoupe donc, dans le cas de la presse quotidienne, celle de la capacité de publics donnés à apparaître sous une forme politique reconnaissable (Butler, 2016). Dans la présente controverse, la mobilisation contre Exhibit $B$ est très majoritairement disqualifiée par la

3 La requête sera déboutée le mardi 9 décembre 2014. Le collectif saisira ensuite sans succès le Conseil d'État.

4 Les quarante-et-un articles du corpus se répartissent de la façon suivante : Libération (18 articles), Le Monde (9), Aujourd'hui en France (6), L'Humanité (5), La Croix (2), Le Figaro (1). 
presse, par le biais d'une représentation de ses acteurs comme incompétents en matière de jugement esthétique et excessifs sur le plan politique. En tant que scène d'apparition, la presse est un lieu de régulation de la visibilité. Dans le cas étudié, l'apparition prend cependant la forme, en apparence paradoxale, d'une disparition contrôlée, d'une visibilité sur le mode de la spectralité, d'un discours sur la mobilisation qui organise le silence de ses principaux acteurs. La médiation journalistique de la controverse est placée sous le signe de la partition raciale, sensible dans le déséquilibre des voix appelées à exposer le racisme dans les colonnes des quotidiens. Rares sont les personnes s'identifiant comme noir·e·s à avoir été invité·e·s à partager leur position au sein de la presse nationale quotidienne (que ce soit dans des tribunes ou par des propos rapportés); et une seule personne directement engagée dans la mobilisation signe une intervention. C'est donc en tant que scène de disparition que la presse quotidienne nationale sera ici comprise : ce qui se joue dans le cadrage médiatique de la controverse étant moins de l'ordre d'un débat sur la qualité raciste ou antiraciste de l'œuvre de Brett Bailey que d'un partage inégal de la visibilité entre différentes définitions du racisme.

\section{1. Incompétence esthétique}

Fondé sur une " esthétique [du] choc " (Libération, 21 novembre 2014), le dispositif théâtral conçu par Brett Bailey viserait, selon l'avis quasi unanime de la presse, à " donner conscience » (Le Monde, 29 novembre 2014). La remise en cause de l'œuvre porte toutefois précisément sur le type de conscience qu'il s'agirait ici d'éveiller. La pétition qui a recueilli plus de 20000 signatures, initiée par John Mullen et demandant une déprogrammation, la présente comme " une insulte à ceux et celles (dont une bonne partie des habitants où est programmée l'exposition) qui se trouvent bien obligés de comprendre le racisme parce qu'ils le subissent quotidiennement ${ }^{5}$. Bien qu'elle constitue le point de départ le plus saillant de la mobilisation, la pétition est rarement citée dans la presse et, lorsqu'elle l'est, ne fait l'objet que de simples évocations. Cette pétition porte avant tout un questionnement sur l'adresse de l'œuvre : à qui est donc destiné le choc sensible du spectacle de la violence raciste ? Le public imaginé du dispositif ne serait-il pas fondé, en vertu de ce principe d'éveil d'une conscience antiraciste, sur l'exclusion des " premiers concernés " (Le Monde, 29 novembre 2014) ? Ou, comme l'explicite l'auteure afroféministe B. K. Lomami dans Libération (2 décembre 2014), "Qu'est-ce que cette installation est censée apporter aux Noir(e)s ? S'en soucie-t-on ?».

5 Mullen J. (2014). «Déprogrammer le zoo humain ». In Change.org. Disponible sur : https://www.change.org/p/centre-104-th\%C3\%A9\%C3\%A2tre-g\%C3\%A9rard-philipe-d\%C3\%A9programmer-le-zoo-humain-exhibitb-contrexhibitb (page consultée le 4 septembre 2016). 
La critique ici formulée à l'encontre d'Exhibit $B$ se situe distinctement à l'articulation du politique et de l'esthétique, entrant en résonnance avec la conception de cette jonction que donne Jacques Rancière $(2000,12)$ au travers du concept de "partage du sensible ", ce "système d'évidences sensibles qui donne à voir en même temps l'existence d'un commun et les découpages qui y définissent les places et les parts respectives ». Le journaliste du Monde Michel Guerrin refuse toutefois de voir dans la mobilisation contre Exhibit $B$ un quelconque fondement esthétique : "ce rejet [de l'œuvre de Bailey], écrit-il, n'est pas tant esthétique que communautaire » (6 décembre 2014). La connaissance du racisme revendiquée par les manifestant·e.s mobilisé·e·s contre la pièce - qui s'affirme dans la pétition précitée tout autant que sur les pancartes que l'on a pu voir agitées sur le parvis du TGP de Saint-Denis ${ }^{6}$ - se trouve requalifiée en incompétence esthétique.

Cette incompétence se situe à deux niveaux. Elle s'exprimerait d'abord, selon la presse, dans le fait que les manifestant.e.s « confondr[aient] la représentation et son objet "(L'Humanité, 8 décembre 2014). Cette confusion entraînerait une désorientation politique, à laquelle plusieurs articles tentent de remédier 7 . Interviewée dans Libération (2 décembre 2016), l'avocate Agnès Tricoire, qui s'exprime pour la LDH, regrette qu'« au lieu de demander des comptes au pouvoir politique, on s'attaque à un objet symbolique qui dénonce le même mal ${ }^{8}$; tandis que le journaliste Laurent Carpentier évoque dans Le Monde (28 novembre 2014) ces jeunes de Saint-Denis " capuches relevées, moteur du scoot à l'arrêt, le trois-feuilles à moitié roulé, [qui] se préoccupent plus de la misère dont est fait leur quotidien que de la question de sa représentation ». La récurrence de ces injonctions à une réorientation politique peut étonner, tant elle donne la nette impression d'une dépolitisation du théâtre. Toutefois, il ne s'agit pas tant de dissocier l'esthétique théâtrale du politique que de distribuer inégalement la légitimité politique entre l'artiste et les publics. Les "motivations " de Brett Bailey sont bien décrites dans Libération comme « politiques » (21 novembre 2014) et évaluées sur ce même plan comme " parfaitement correcte[s]» (24 novembre 2014). Certains articles soulignent clairement que c'est l'incompétence esthétique des « anti-Exhibit $B$ » qui serait source de tensions politiques. Le journaliste Michel Guerrin (Le Monde, 6 décembre 2014) écrit par exemple :

7 "Qu'on ne se trompe pas de cible », écrivent par exemple Michèle Riot-Sarcey et Claudia Moatti (Libération, 11 décembre 2014). "L'indignation ne devrait pas être tournée contre cette performance, mais contre ceux qui ignorent le lien entre la condition des esclaves et celle de l'immigrant moderne ". On s'amusera de constater que la " cible » à laquelle renvoie préférentiellement les auteures est précisément le public type que la majeure partie de la presse imagine être celui de l'œuvre de Bailey : un public « ignorant ».

8 Elle ajoute que «le point commun [de la mobilisation contre Exhibit B] avec la rhétorique d'extrême droite, c'est de dénier à l'art son statut de représentation, de l'assimiler à du discours littéral ». 
Pour l'avocat Emmanuel Pierrat, " on peut beaucoup moins montrer d'œuvres audacieuses qu'il y a trente ans. Parce que ces œuvres sont vues sur Internet par un public à qui elles n'étaient pas destinées ». Internet réussit là où trente ans de politiques culturelles ont échoué : attirer un public qui pense que tel spectacle n'est pas pour lui.

Le danger de la décontextualisation ici identifié semble résider dans le fait d'exposer certains publics à certains contenus ${ }^{9}$. L'enjeu serait donc celui d'un contrôle strict de la circulation des représentations. Tout se passe comme s'il s'agissait de réserver la représentation théâtrale (ou cette représentation théâtrale ?) à des publics déterminés, dont semblent ne pas faire partie les militant-e.s anti-Exhibit $B$. La question initiale posée par John Mullen revient alors avec force : si ce n'est pas à ce public que l'œuvre était destinée, alors à qui ? Comme l'affirme Olivier Neveux $(2013,8)$ :

Au théâtre, ce qui est politique (une politique conflictuelle, de rupture, de négation, d'émancipation) est la conception implicite ou explicite, spontanée ou théorisée, que le spectacle porte de son spectateur, le "spectateur » qu'il construit (ou non) et le rapport qu'il entend nouer avec lui.

Aussi, comment comprendre la dimension politique affichée de la machine représentationnelle d'Exhibit $B$ si, comme le montre la médiation journalistique de la controverse, celle-ci tourne à l'exclusion?

L'incompétence esthétique des militant·e·s se manifesterait ensuite dans le fait qu'ils n'auraient pas vu l'œuvre, comme l'écrivent Jean Bellorini et José-Manuel Gonçalvès, les directeurs des deux établissements culturels concernés, dans un communiqué de presse commun : "Les auteurs de cette pétition reconnaissent ne pas avoir vu l'installation-performance. Comment peut-on juger une œuvre sans l'avoir vue et demander son annulation sans la connaître et en l'accusant du contraire de ce qu'elle dénonce ? $»^{10}$. Assimilant la contestation d'Exhibit $B$ à la mobilisation de groupuscules d'extrême droite et catholiques contre les pièces Golgota Picnic de Rodrigo Garcia et Sur le concept du visage du fils de Dieu de Romeo Castellucci en 2011'11, le journaliste

9 On retrouve cet argument dans un article du Monde (24 février 2015) signé Raphaëlle Bacqué, dans lequel elle liste les menaces contre la liberté d'expression dans la " France post-'Charlie' » (article qui situe la contestation contre Exhibit $B$ dans un historique hétérogène comprenant par exemple les attentats commis par des groupuscules catholiques contre le film de Martin Scorcese, La dernière tentation du Christ). On peut y lire, à propos des caricatures notamment publiées dans Charlie Hebdo : "Les dessins d'un petit journal satirique 'sont devenus accessibles à des populations qui ne peuvent pas les comprendre', observe l'essayiste Caroline Fourest » (mes italiques).

10 Le communiqué, intitulé "Le débat oui, la censure non ", a été rendu public le 18 novembre 2014. Il est notamment cité dans La Croix (24 novembre 2014).

11 Les militants de l'Action française, du Renouveau français et du GUD mobilisés contre ces pièces revendiquaient de ne pas les avoir vues. À propos des contestations à l'encontre de la pièce de Romeo Castellucci, voir Cervulle (2012). 
Laurent Carpentier écrit quant à lui dans Le Monde (29 novembre 2014) : « partout la même chose : on n'a pas vu, on ne veut pas voir, on sait : c'est honteux, c'est dégoutant, il faut que ça cesse ! " (mes italiques). Au-delà de ravaler au même rang - sans hiérarchie politique aucune - le niveau du racisme et des atteintes aux chrétiens dans la France contemporaine, cette position renvoie surtout fermement ceux et celles qui refusent d'occuper la place d'ignorants dans le dispositif de Bailey... à une position d'ignorance, cette fois due à leur méconnaissance de l'œuvre.

Cette représentation des manifestant.e-s et pétitionnaires tend à les exclure radicalement de la catégorie de public, qui apparaît définie par le fait d'avoir vu l'œuvre. Une telle définition occulte cependant le fait que le choix explicite de refuser de voir une œuvre constitue en soi une forme de réception; en ce sens il s'agit bien là d'un public, que l'on peut qualifier d'oppositionnel. Ce public ne refuse pas absolument le spectacle mais, plutôt, le considère depuis un autre point de vue, plus oblique, qui inclut dans son champ les publics ordinaires de l'installation-performance et la manière dont la médiation de l'œuvre les constitue comme tels. La notion de "public oppositionnel » ici esquissée s'appuie, en l'élargissant, sur celle de « regard oppositionnel », définie par bell hooks (1992, 115-132). Hooks décrit ainsi le type de regard critique développé par les spectatrices noires, exclues du public implicite de la culture visuelle prédominante, et tend à affirmer un droit de regard. Le cas d'Exhibit $B$ et des " injonctions à voir » qui se sont multipliées durant la médiatisation de la controverse montre parfaitement combien ce « regard oppositionnel » peut parfois impliquer de regarder non seulement autrement, mais aussi ailleurs. Comme l'écrit Michael Warner $(2014,12)$, "I'orientation de notre regard participe de la constitution de notre monde social ». Un type de regard - caractérisé par un point de vue et une intensité donnés - signale l'appartenance à une arène discursive, où peut être articulée une certaine conception du monde social.

Le public oppositionnel d'Exhibit $B$ tel que je le conçois n'est ainsi pas très éloigné des « contre-publics subalternes » que décrit Nancy Fraser $(2001,138)$, qui « élaborent et diffusent des contre-discours, afin de formuler leur propre interprétation de leurs identités, leurs intérêts et leurs besoins ». L'un comme les autres sont situés en opposition à ce que Fraser appelle la « sphère publique officielle » et les " publics dominants ». Je souhaite toutefois insister particulièrement sur l'intrication de la question de la visibilité avec celle du regard, assez marginale dans l'analyse de Fraser. La capacité d'une mobilisation à être visible repose, selon Judith Butler $(2016,55)$, sur « une structuration régulatrice du champ de l'apparition qui définit qui peut être vu, entendu et reconnu ». On peut penser que parmi les conditions de l'apparition dans la sphère publique officielle se trouve la nécessité pour ladite mobilisation de voir certains objets, de les voir depuis un certain point de vue et avec une certaine intensité ; dit autrement, pour qu'une parole soit pleinement perçue comme légitime, elle doit donner des gages de reconnaissance de certains types de problèmes, construits selon une position déterminée et investis d'affects particuliers. 
La controverse autour d'Exhibit $B$ a ceci d'intéressant qu'elle rend littérale la métaphore du regard : la visibilité du public oppositionnel dans la sphère publique officielle est directement conditionnée à la vision de l'installation-performance. En ce sens, c'est un certain type de regard, une manière de voir ou en l'occurrence de ne pas voir, qui est discréditée par la presse. Pour cette dernière, la controverse porte sur ce qu'il y a à voir dans l'œuvre de Bailey, tandis que pour le public oppositionnel, l'enjeu réside dans la politisation de l'acte de ne pas voir, c'est-à-dire dans le refus de l'institutionnalisation d'un certain regard antiraciste.

\section{2. \\ Excès politique}

À lire les chroniques de la controverse et les prises d'opinion publiées dans la presse, il semblerait que l'œuvre de Bailey achoppe sur ce qui constitue justement son projet initial : celui de mettre les publics en mouvement, d'activer en eux un potentiel politique par la mise en connexion du passé et du présent de la violence raciste. N'est-ce pas là exactement ce que font les publics contestant l'œuvre, au point où la politisation de l'activité de public fait déborder le théâtre hors de ses murs et entraîne un refus pur et simple de la place assignée au spectateur par l'œuvre? Pourquoi, ainsi, ne pas voir dans la mobilisation contre Exhibit $B$ non pas la sortie de " mauvais " publics, frappés d'incompétence esthétique et donc politique, mais au contraire le public " idéal », que l'artiste entendait précisément générer ? Dit autrement, pourquoi ne pas considérer, en adoptant la grille de lecture de Bailey lui-même ${ }^{12}$, que la mobilisation serait moins le signe d'un échec que d'une réussite artistique?

La disqualification de la mobilisation, plutôt que sa saisie pour réaffirmer, par exemple, la puissance politique du théâtre, provient sans doute de l'ambiguïté même dans laquelle se trouve l'œuvre de Bailey. On peut en effet voir en elle une tentative de conjuration de ce que Rancière appelle le " paradoxe du spectateur » $(2008,8)$. S'il n'est de théâtre sans spectateur, ce dernier fut cependant renvoyé, tout au long de l'histoire du théâtre et des critiques qui ont pu lui être opposées, à une condition d'ignorance et de passivité. Les réponses à ces critiques ont soit tenté de faire des spectateurs des " participants actifs au lieu d'être des voyeurs passifs » (Rancière, 2008, 10), soit ont au contraire

12 On apprend sous la plume de Sabine Cessou, dans Libération (8 décembre 2014), que la controverse aurait « plongé [Brett Bailey] dans un mélange 'd'empathie, de tristesse et de colère'. Empathie avec les manifestants dehors, face à un fort déploiement policier. 'Mon art défend ce qu'ils défendent', soutient-il ». En effet, la mobilisation semble parfaitement incarner la visée même de l'œuvre telle qu'il la décrit dans ce même article : "'Faire exploser de l'intérieur, par le regard et la dignité, le stéréotype du corps noir passif et victime' ». 
tendu vers une critique de la médiation théâtrale elle-même, ne voyant dans le théâtre qu'un « lieu où des ignorants sont conviés à voir des hommes souffrants » (Rancière, 2008, 9). L'installation-performance de Bailey semble se situer à mi-chemin de ces deux voies : elle oppose la mobilité des spectateurs/trices à la stase des acteurs/trices, tout en envisageant la représentation naturaliste de la souffrance extrême comme une voie d'accès à la connaissance. Or ces deux conceptions implicites de la place du spectateur contribueraient selon Rancière, plutôt que de l'émanciper, à l'enfermer à double tour dans cette ignorance et cette passivité que l'artiste prétend exorciser. Mais n'est-ce pas " justement la volonté de supprimer la distance [entre l'activité et la passivité, entre la connaissance et l'ignorance] qui crée la distance »? (Rancière, 2008, 18). Selon une telle lecture, le public oppositionnel apparaîtrait moins comme marqué par le déficit (ignorance, passivité) que par un excès de connaissance et d'action rendant la médiation théâtrale de Bailey et sa pensée implicite du spectateur caduques.

C'est également par cet excès, qui outrepasse les formes contrôlées du politique et de l'esthétique, que le public oppositionnel d'Exhibit $B$ est caractérisé dans la presse. La forme prise par l'expression politique de ce public est, en particulier, l'objet de toutes les attentions. Celle-ci est présentée comme non seulement mal orientée (donc illégitime), mais disproportionnée dans son intensité. De telles modalités de caractérisation de l'expression politique ont pour effet, en délégitimant son objet aussi bien que ses moyens, d'exclure ceux et celles qui la portent du champ politique pour les confiner au domaine de la violence (Donegani et Sadoun, 2003, 3-4). "Pourquoi tant de violence, écrivent par exemple dans Libération (11 décembre 2014) les historiennes Michèle Riot-Sarcey et Claudia Moatti, contre l'artiste, contre le théâtre et contre les visiteurs, accusés de voyeurisme? ». La " contestation » est présentée comme « brutale » dans Aujourd'hui en France (10 décembre 2014) ; elle brandit, peut-on lire dans Le Monde (6 décembre 2014), « les armes de l'intimidation, de l'insulte, du coup de poing ". Si cette insistance sur la violence renvoie aux incidents survenus lors de la deuxième journée de manifestation devant le TGP - où l'une des vitres de l'établissement fut brisée - force est de constater que ce registre de disqualification chevauche parfois celui de la racialisation, certains articles renvoyant de ceux et celles qui militent contre le spectacle de Noirs en chaînes l'image d'une foule " noire » " déchaînée » (Aujourd'hui en France, 10 décembre 2014). Au-delà, c'est même l'ombre de la figure du «terroriste " qui plane, les militant·e.s contre Exhibit $B$ étant assimilé.e.s aux « fondamentalistes religieux, nationaux ou ethniques » (Libération, 17 septembre 2015), voire intégré·e·s à I'historique des menaces contre la liberté d'expression dressé par Raphaëlle Bacqué pour Le Monde (24 février 2015).

La qualification d'un geste politique comme violent ne peut se comprendre qu'en référence à une forme mesurée et modérée du politique. Cette dernière est incarnée dans la presse par une position universaliste qui constitue le site d'énonciation partagé de la grande majorité des journalistes et intellectuel·le·s 
qui s'y expriment à l'occasion de la controverse. L'anthropologue Jean-Loup Amselle oppose ainsi dans Libération (17 septembre 2015) «l'universalité de l'art » à un particularisme en vertu duquel « se met[trait] en place une sorte de censure sélective qui fait que chaque groupe prêche en quelque sorte pour sa paroisse et ne défend que ses propres valeurs ". De son côté, l'historien Pascal Blanchard met en tension, dans Le Monde (29 novembre 2014), la dimension " universelle » de l'histoire des zoos humains et la " communautarisation des mémoires " dont la mobilisation contre Exhibit $B$ serait selon lui l'indice. La référence à l'universalisme sert, la plupart du temps ${ }^{13}$, à draper la mobilisation antiraciste non seulement des oripeaux du communautarisme, mais du racisme. On accuse en particulier les manifestant·e·s d'entraver la liberté d'expression d'un artiste en raison de la couleur de sa peau. Comme l'écrit la journaliste Marie-José Sirach dans L'Humanité (1er décembre 2014), le metteur en scène " est blanc... lui reprochent certains des opposants au spectacle ». Le réalisateur Nicolas Klotz affirme de son côté, dans une tribune parue dans Libération (5 décembre 2014), que " reprocher à Brett Bailey d'être blanc et de travailler avec des Noirs est d'une connerie crasse ". Ces positions s'appuient - sans la citer - sur un passage de la pétition de Mullen :

Il est déjà surprenant que dans des quartiers mixtes au Nord de Paris, on invite la population multiethnique à venir apprendre sur le racisme d'un Sud-Africain blanc. C'est d'autant plus choquant que les possibilités pour des artistes noirs de présenter leur œuvre dans des centres culturels prestigieux sont extrêmement limitées.

Le cœur du propos ne porte ici pas tant sur l'identité du metteur en scène $d^{\prime}$ Exhibit $B$ que sur le fait que sa programmation joue comme un révélateur de cet " océan blanc " (L'Humanité, 1er décembre 2014) qu'est, en France, le monde de la culture. Rares sont toutefois les articles qui, à l'instar de la tribune du dramaturge et metteur en scène Alain Foix dans L'Humanité (1er décembre 2014), reprennent les revendications qui animent la mobilisation :

Une jeune femme, écrit-il, m’apporte la réponse [au pourquoi de la crispation] sous forme de questions : " [...] Pourquoi jamais des œuvres d'auteurs et d'artistes noirs contemporains ? Pourquoi n'y a-t-il pas de directeurs de théâtre noirs ? Pourquoi n'y a-t-il que des Blancs dans les salles, sur la scène, dans les bureaux et des Noirs à la sécurité ? ".

Aussi la réduction de la critique des anti-Exhibit $B$ à la seule identité de Bailey contribue-t-elle à entretenir la cécité face aux multiples mécanismes

13 Éric Fassin opère dans Le Monde (29 novembre 2014) un geste inverse, s'appuyant sur la nécessité d'une universalité de l'art pour affirmer la légitimité de la mobilisation. "On aurait tort, écrit-il, de réduire ces critiques au communautarisme : il ne s'agit pas de prétendre que les Noirs seuls pourraient parler des Noirs, mais au contraire de revendiquer que l'art doit pouvoir s'adresser à toutes et tous à la fois ». 
d'exclusion, voire à la division raciale du travail, qui sous-tendent l'organisation du théâtre public en France. La tentative de constituer cet enjeu en problème public se voit majoritairement discréditée dans la presse, au travers d'une part d'une occultation pure et simple de ce niveau de revendication et, d'autre part, d'un processus d'inversion rhétorique faisant porter l'accusation de racisme sur ceux et celles-là mêmes qui en dénoncent la dimension systémique (Ahmed, 2012 ; Dalibert, 2014).

\section{3. Mains blanches}

Dans la mobilisation contre Exhibit $B$, deux manières de donner à voir le racisme s'affrontent. La première stratégie de représentation, qu'incarne I'œuvre de Brett Bailey, invite à parcourir des scènes de souffrance ; elle conçoit le théâtre comme un lieu d'abolition de la fausse conscience que génère la "ligne de partage des couleurs " (Du Bois, 2007, 20). Ce qui semble toutefois manquer au tableau - comme le soulignait la pancarte d'une manifestante devant le TGP - ce sont « les mains blanches qui [...] ont mis [les Noir.e.s] en cage ». Interrogé dans Libération (8 décembre 2014) sur l'absence de corps blancs dans l'installation, Brett Bailey répond que « les Blancs n'ont jamais été les objets déshumanisés d'un tel racisme systématique ", mais n'envisage à aucun moment leur représentation sous la forme de "racisants " - pour reprendre le terme de Colette Guillaumin $(2002,18)^{14}$. Sans doute car le dispositif repose sur la présence de spectateurs/trices blanc.he-s pour occuper cette fonction (sinon quelle signification prêter au fait que les acteurs/trices noir.e.s objectivé·e·s renvoient au spectateur un regard de réprobation, de résistance et de dignité) $)^{15}$ ?

L'œuvre qui prétend éveiller la conscience de publics ignorant l'actualité du racisme historique feint elle-même d'ignorer qu'elle doit s'appuyer pour ce faire sur une économie plus large de l'exclusion : celle d'une homogénéité relative des publics voire, pour un centre dramatique comme le TGP de Saint-Denis, d'une implantation imparfaite dans le territoire ${ }^{16}$, mais aussi celle

14 On pourrait ajouter que Bailey méconnait l'effet des techniques de déshumanisation sur ceux-là mêmes qui les mettent en œuvre. Comme l'écrit Aimé Césaire $(1955,18)$ : « Le colonisateur qui, pour se donner bonne conscience, s'habitue à voir dans l'autre la bête, s'entraîne à le traiter en bête, tend objectivement à se transformer lui-même en bête ".

15 Dans son étude détaillée de l'œuvre, Marc Maire $(2016,626)$ souligne par ailleurs que "Brett Bailey a toujours affirmé que sa trilogie Exhibit était d'abord destinée aux Blancs».

16 Le directeur du TGP entre 2008 et 2013 , Christophe Rauck, indique dans ses réponses au rapport d'observations de la Cour des Comptes que «l'existence d'un centre dramatique national à Saint-Denis, ville populaire et multiculturelle, s'inscrit de fait dans le double projet des pionniers de la décentralisation 
de la réservation des fonctions de programmation, définition et mise en œuvre de la politique culturelle. Cette stratégie de représentation se trouve dès lors porteuse d'une conception tout à fait étriquée du racisme, limitant son champ d'application au seul domaine idéologique, voire moral, prétendant percer au travers de ce médium qu'est la race et qui recouvre nos yeux d'un filtre de perception (Mitchell, 2012). Dans un contexte de programmation français, une telle stratégie de représentation se trouve aisément habilitée et autorisée, d'abord parce qu'elle n'interroge pas l'organisation sociale même, ensuite et surtout parce qu'elle apparaît formulée depuis une position d'énonciation immédiatement reconnaissable : celle du corps national (incarné par les établissements culturels) tâchant d'évacuer de son sein les scories d'un temps que l'on tient pour révolu. Le racisme n'apparaît là que sous la forme d'un résidu, trace à demi effacée qu'une ultime prise de conscience - ici envisagée par la médiation théâtrale - devrait permettre de faire disparaître à jamais. Pourtant, en s'adressant au public par la mise en spectacle d'une culpabilité historique, elle contribue à construire son spectateur sous une forme essentialisée, l'interpellant « dans son identité européenne, dans son identité blanche, bref dans son 'fardeau d'homme blanc' » (Larcher, 2015, 227).

La seconde stratégie de représentation, celle du public oppositionnel $\mathrm{d}^{\prime} E x$ hibit $B$, ne consiste pas à mettre en lumière l'ignorance (du racisme) de certains publics, mais le processus de construction sociale de cette ignorance ${ }^{17}$ auquel participe aussi bien l'œuvre que son contexte de programmation. Ce sont ainsi les modalités d'inscription sociopolitique et le contexte institutionnel de déploiement de l'œuvre qui sont interrogés et soumis à la critique. Seul le refus du mode de production et de médiation de l'œuvre permet de comprendre véritablement le refus de la voir. L'enjeu, en effet, ne se situe pas dans l'évaluation de la pertinence politique de la représentation que l'œuvre donne du racisme mais, plutôt, dans la mise en cause d'une politique culturelle dont l'aveuglement face au partage racial autorise justement une définition étroite du

dramatique (démocratisation et régionalisation de la création théâtrale) dans le sens qu'il propose une offre artistique et culturelle dans un territoire où une partie très importante de la population ne fait pas partie des groupes sociaux qui fréquentent le théâtre et ce pour des raisons sociales, géographiques, culturelles ou financières » (Rauck, 2012, 2). Une enquête sur les publics du TGP conduite par Christine Bellavoine en partenariat avec l'établissement relève que seuls $25 \%$ de ses publics habite à Saint-Denis. Si l'on retient ensuite le seul critère de la catégorie socioprofessionnelle, les cadres et professions intellectuelles supérieures représentent $40 \%$ de la fréquentation du TGP, les professions intermédiaires $23,5 \%$, les employé.e.s $15 \%$, les ouvrier.e.s $2,5 \%$ (Bellavoine, 2015). Les données statistiques de l'INSEE (2013) permettent de mettre en relief ces chiffres avec la répartition de la population de Saint-Denis selon la catégorie professionnelle : 7,6 \% des dionysiens de plus de 15 ans sont cadres ou relèvent des professions intellectuelles supérieures, 12,7\% sont assimilables aux professions intermédiaires, 23,5 sont employé·e.s et $17,1 \%$ sont ouvrier.e.s.

17 Sur la fonction de l'ignorance dans la subjectivation blanche, voir Cervulle (2013). 
racisme, qui la conforte dans ses orientations. La conception du racisme portée par la mobilisation contre Exhibit $B$ souligne la profonde inscription du racisme dans les structures idéologique, économique et politique (Bonilla-Silva, 1996). Identifier le racisme selon une telle conception systémique pose toutefois une certaine difficulté, du point de vue du lieu de l'énonciation : comment se situer hors de ce qui n'a pas de dehors, comment dégager une position d'extériorité autorisant un retournement de la pensée contre cela même qui la constitue, la traverse et la rend possible ? Cette contradiction - dans laquelle se trouve également prise, sur un plan épistémologique, la critique décoloniale (Dell'Omodarme, 2016) - se manifeste avec éclat dans le cas présent. L'antiracisme qui porte une conception systémique du racisme est particulièrement vulnérable à la disqualification en raison du trouble énonciatif dans lequel il semble se trouver, du dehors qu'il laisse entrevoir mais qui ne saurait advenir pleinement sans une profonde remise en cause du contexte depuis lequel il émerge. La contradiction par laquelle cet antiracisme radical parle du présent depuis la position d'un futur à construire (selon un mécanisme propre aux pensées critiques organisées par une utopie $\left.{ }^{18}\right)$, devient l'instrument d'une disqualification.

En effet, ce que montre la controverse autour d'Exhibit $B$ telle qu'elle se construit dans la presse quotidienne, c'est que le monde de la culture (journalistes, intellectuel·le·s, artistes et directeurs d'établissements culturels) ne sait que faire des voix de ce public qui s'exprime depuis une position à leurs yeux paradoxale : celle d'une critique de l'institution depuis son dehors, prenant pour objet non ce qui « devrait » être discuté (la valeur esthétique et la signification des œuvres), mais la programmation elle-même et la logique institutionnelle de construction des publics dont elle est le reflet. Les contestataires rassemblé.e.s devant le TGP et le Centquatre composent un public qui d'une certaine façon, d'un point de vue institutionnel, ne pourrait ni ne devrait exister comme tel. Quand a-t-on vu les "publics éloignés ", selon le vocable euphémisant de la médiation culturelle, se rapprocher d'un établissement pour lui reprocher de produire de la distance ? Pour politiser l'éloignement en le requalifiant comme exclusion ? Ce que ce public oppositionnel expose ainsi, c'est « la symbolique de la séparation » qui fonde la médiation culturelle; organisant une véritable " altérité de l'intérieur », pour reprendre les termes de Nacira Guénif-Souilamas $(2007,347)$. Pensée sous la forme d'un écart à réduire ou d'un vide à remplir, la médiation culturelle s'élève sur une figure de l'extériorité ("publics éloignés ", voire "empêchés ») dont la fonction est de sécréter une certaine idée de l'intériorité, c'est-à-dire une certaine manière d'habiter l'espace de la culture. Or, cette figure est le produit d'un point de vue de l'intérieur des murs de l'institution sur cela même qui donne sens à sa mission ("l'accès à la culture » et sa

18 Tout au moins lorsque cette utopie tend « à exposer la contradiction sociale plutôt que de la neutraliser, à troubler l'homologie entre corps individuel et corps social plutôt que de la soutenir »(Burwell, 1997, 205 cité dans Chan, 2006, 467). 
" démocratisation »). C'est en ce sens qu'il s'agit d'une altérité de l'intérieur, car ce public que l'on représente comme éloigné hante nécessairement les lieux culturels et leurs logiques de fonctionnement, sur le mode paradoxal d'une absence présente. Cependant, comme le souligne Guénif-Souilamas (2007, 347), « plus l'altérité est utile à la définition de l'intériorité, plus elle est condamnée à être calcifiée, matérialisée et réifiée dans cette fonction d'extranéité et d'altérité ". D'où sans doute l'insistance, perceptible dans la presse, à renvoyer le public oppositionnel $d^{\prime} E x h i b i t B$ dans une relation antinomique avec les formes prescrites de l'esthétique et du politique.

\section{4. Doublure}

"'Décolonisons l'imaginaire !' pouvait-on lire sur une banderole des anti-Exhibit B. Surtout pas. Si chaque communauté s'érige en commissaire du peuple, des milliers de livres pourraient être brûlés, des expositions fermées ". Ces mots de Michel Guerrin dans les colonnes du Monde (6 décembre 2014) permettent d'identifier l'espace de conflictualité ouvert par la controverse. Ces propos illustrent parfaitement combien l'actualité véritable d'un projet de décolonisation - qui ne serait pas contenu dans le strict domaine de la représentation, mais qui remettrait en question les conditions d'existence du représentable et du représenté - est perçue comme la marque d'une violence. Aussi le terrain réservé de la culture ne serait-il pas à décoloniser, "surtout pas "... Dans le sillage de la mobilisation contre Exhibit $B$ ont toutefois émergé des initiatives collectives visant à établir durablement certaines des voix composant ce public oppositionnel. Parmi celles-ci se trouve le collectif Décoloniser les arts, regroupant artistes et professionnels des arts et de la culture, qui a notamment publié en février 2016 une lettre ouverte demandant aux responsables de théâtres, festivals et établissements culturels de s'interroger sur le "manque de représentation des populations 'non-blanches' sur les plateaux » et appelant à la mise en place d'outils de mesure statistique permettant de quantifier l'ampleur de la partition raciale au sein de ce champ professionnel ${ }^{19}$.

La médiatisation de la controverse se trouve elle-même organisée par une telle partition raciale, qui s'exprime notamment dans la relative absence de mise en interrogation du paysage culturel français en tant que sous-tendu - au même titre que tout autre espace du monde social - par le racisme systémique, y compris et peut-être même précisément dans le cas d'une « œuvre artistique

19 Décoloniser les arts (2016). « Lettre aux directeurs et directrices de théâtre, ou de festivals, lettre aux responsables culturels ». In Mediapart. Disponible sur : https://blogs.mediapart.fr/decoloniser-les-arts/blog/240216/lettre-aux-directeurs-et-directrices-de-theatres-ou-de-festivals-lettre-aux-responsables-c (page consultée le 4 septembre 2016). 
officiellement antiraciste » (Larcher, 2015, 222). La représentation médiatique de la mobilisation contre Exhibit $B$ constitue ainsi la doublure, cousue de fils blancs, de la représentation théâtrale de Brett Bailey. Dans un cas comme dans l'autre, le véritable niveau de disqualification du public oppositionnel se situe moins dans la contestation de sa connaissance (du racisme), de sa sensibilité (esthétique) et de son action (politique) que dans l'occultation quasi complète de ces dernières. Les voix de ce public n'existent dans la presse qu'à l'état spectral : réduites à des abstractions dont on peut même, comme le préconise Marie-José Sirach dans L'Humanité (1er décembre 2014), "faire abstraction ». Toutefois, même le silence qu'organisent les pages des journaux et les scènes de théâtre peut, pour qui sait l'entendre, couvrir de son intensité le bruit blanc qui partout assourdit. 
Bibliographie

Ahmed S. (2012). « Les rabat-joie féministes (et autres sujets obstinés) ", trad. O. Bonis. In Cahiers du genre, $n^{\circ} 53$, p. 77-98.

Bellavoine C. (2015). "L'enquête de public(s) du TGP de Saint-Denis ". In Saint-Denis. Au fur et à mesure, $n^{\circ} 64$, p. 23-44.

Bonilla-Silva E. (1996). « Rethinking Racism : Toward a Structural Interpretation ». In American Sociological Review, vol. 62, n³, p. 465-490.

Burwell J. (1997). Notes on Nowhere: Feminism, Utopian Logic, and Social Transformation, Minneapolis, University of Minnesota Press.

Butler J. (2016). Rassemblement. Pluralité, performativité et politique, trad. C. Jaquet, Paris, Fayard.

Cervulle M. (2012). « 'Heureux celui qui croit sans avoir vu'. À propos de la réception de la pièce de Romeo Castellucci, Sur le concept du visage du fils de Dieu ». In Okwui Enwezor et al. (dir.), Intense Proximité. Une anthologie du proche et du lointain, Paris, ArtLys, p. 68-73.

Cervulle M. (2013). Dans le blanc des yeux. Diversité, racisme et médias, Paris, Éditions Amsterdam.

Césaire A. (1955). Discours sur le colonialisme, Paris, Présence africaine.
Chan E. K. (2006). " Utopia and the Problem of Race. Accounting for the Remainder in the Imagination of the 1970s Utopian Subject ». In Utopian Studies, vol. 17, n³, p. 465-490.

Dalibert M. (2014). « Un (anti)racisme légitime dans les médias ? ». In Mouvements, $\mathrm{n}^{\circ} 79$, p. 139-147.

Dell'Omodarme M. (2016). « Anibal Quijano et la saturation coloniale ». In Cervulle M., Quemener N. et Vörös F. (dir.), Matérialismes, culture et communication. Tome 2. Cultural Studies, théories féministes et décoloniales, Paris, Presses des Mines, p. 297-314.

Donegani J.-M., Sadoun M. (2003). «Ce que le politique dit de la violence ". In Raisons politiques, vol. 1, n 9, p. 3-18.

Du Bois W. E. B. (2007). Les âmes du peuple noir, éd. et trad. par M. Bessone, Paris, La Découverte.

Fraser N. (2001). « Repenser la sphère publique : une contribution à la critique de la démocratie telle qu'elle existe réellement ", trad. M. Valenta. In Hermès, $n^{\circ} 31$, p. 125-156.

Guénif-Souilamas N. (2007). « L'altérité de l'intérieur ». In Smouts M.-C. (dir.), La situation postcoloniale, Paris, Presses de Sciences Po, p. 344-352.

Guillaumin C. (2002). L'idéologie raciste. Genèse et langage actuel, Paris, Gallimard.

Hooks B. (1982). Black Looks. Race and Representation, 
Boston, South End Press.

INSEE (2013). « Commune de Saint-Denis (93 066) - Dossier complet ». In Insee.fr, Disponible sur : http://www.insee. $\mathrm{fr} / \mathrm{fr} /$ themes/dossier_complet. asp?codgeo=COM-93066 (page consultée le 4 septembre 2016).

Larcher S. (2015). « Troubles dans la 'race'. De quelques fractures et points aveugles de l'antiracisme français contemporain ". In L'Homme et la société, vol. 4, n²198, p. 213-229.

Maire M. (2016). « La passion, noire et animiste, selon Brett Bailey ». In Cahiers d'études africaines, vol. 3, n 223, p. 607-636.

Mbembe A. (2013). Critique de la raison nègre, Paris, La Découverte.

Mitchell W. J. T. (2012). Seeing through race, Cambridge et Londres, Harvard University Press.

Neveux O. (2013). Politiques du spectateur. Les enjeux du théâtre politique aujourd'hui, Paris, La Découverte.

Rancière J. (2005). Le partage du sensible. Esthétique et politique, Paris, La Fabrique.

Rancière J. (2008). Le spectateur émancipé, Paris, La Fabrique.

Rauck C. (2012). «Réponses du TGP - CDN de Saint-Denis au rapport d'observations définitives du 11/09/2012 ». In Contrôle de la Chambre régionale des comptes d'île-de-France, 12 novembre 2012.
Vergès F. et Mestre C. (2016). «Peau claire et masques noirs. Les luttes anti-colonialistes et féministes de Françoise Vergès ". In L'Autre, vol. 17, n¹, p. 91-105.

Warner M. (2014). Publics and Counterpublics, New York, Zone Books. 